\title{
A Novel Qualitative Identification and Control of Two Degree of Freedom Inverted Pendulum based on Ant colony system
}

\begin{abstract}
In many systems qualitative knowledge of the system behavior is sufficient for introducing a robust and adequately accurate response. This paper, presents a qualitative ant colony based algorithm to solve the model estimation problem in control of nonlinear dynamic systems. In this method, a qualitative model for dynamic systems is introduced as a graph and then the optimal path for shortest control time is obtained with the aid of ant colony system (ACS) optimization procedure. This method is well examined by a complicated two degree of freedom (2DOF) inverted pendulum system and the responses are presented. The presented approach has many advantages. First, it increases the robustness of the controlled system. Furthermore, it creates simple implementation through a single look up table (LUT). As a final point, it will be achieved control rule base generation for artificial intelligence methods such as fuzzy logic controller.
\end{abstract}

Alireza Rezazade

Keywords - Ant colony system, Identification, Monte Carlo, Qualitative Control, Graph model.

\section{INTRODUCTION}

Robust control of dynamic discrete time systems is very important in modern control theory. Most of physical systems are described as nonlinear function of time and state of the operation. It is evident that nonlinearity and sensitivity are two important aspects in the analysis of those systems. This makes the control to deviate from the desired operation conditions. Another theme that forces the designer to deal with the systems qualitatively is the limitation on the accuracy of the sensor. In most applications, we need accuracy only in near of target response and during transient, it is not vital to sense the states in high precision. Qualitative identification and control of system, is a method to observe the system behavior in the next time step; when a special amount of excitation is applied to the system [7], [8], [9]. The Qualitative system identification may also play the role of expert in heuristic methods of control like fuzzy controllers. The critical key in fuzzy rule generation in fuzzy logic controllers is the expert information about the system behavior.

Qualitative description of the system behavior is an efficient way of finding the rule bases of the system that can

Manuscript received February 17, 2009. This work was supported in part by the Research management department of Shahid Beheshti University in the framework of the research support Program No.600/2966

A.Rezazade is with the Department of Electrical and Computer Engineering, Shahid Beheshti University, G. C, Evin, Tehran, Iran, 1983963113 (corresponding author to provide phone:+982177538160; fax:+982177538161) be used in heuristic control strategies [9].The first step of the method is weighted quantization, which splits the state space of the desired system to special sections. The sectioning of the state space must be made such that in desired points the resolution of state observation meets the designer conditions. In such cases, it is recommended that the sections become finer around the set points. Each section must be numbered. The second step is to split the input space to quantized values. In many systems such as electromechanical drive systems and switch mode power supplies, the input of the system has finite levels of voltage that is supplied by voltage source inverters. This is because of lowering the power losses in the drivers [3], [4]. Another type of control systems that use quantized input space are the bang-bang or sliding mode control systems that are used in many systems. The main advantage of such control systems is the easy implementation process of such controls due to specified levels of input signals. Such control strategies are widely used in valve operation systems, electromechanical, thermal systems and nonlinear systems [13], [14], and [15]. The next step is to find out the state transition from one quantized state to others in application of all of quantized inputs. The result of the process is a graph that represents the actual system qualitatively. The resulting graph is the qualitative representation of the dynamical system and it must be noted that all system information is hidden in this graph. The expert information can be extracted easily from the maintained graph. The fuzzy expert rules such as the sentences like:" if $\mathrm{X} 1$ is very high and $\mathrm{X} 2$ is medium low and ... and the input $\mathrm{U}$ is high, then the output is Y1" may easily be extracted. The constructed graph represents the dynamical system. In the last step is to find out the best input that transits the system from the present state to the desired state. This can be performed as an optimization procedure. The objective function is complicated and may have many local optimum solutions. The conventional procedures for solving such problem may converge to the locals, so we use heuristic optimization methods in this problem. Ant colony system (ACS) algorithm is used to find an optimal path in this complicated graph for the shortest response time [11], [12], and [16]. The ACS algorithm is mostly fitted to graph optimization problem among the other heuristic optimization algorithms like Genetic algorithm [5], [6]. Finally, the application result of the method to 2DOF-inverted pendulum is presented. The modeling is completely nonlinear and complex [10]. Therefore, the proposed algorithm is implemented on 2DOF-inverted pendulum and the effectiveness of the method is verified. 


\section{GENERAL DESCRIPTION}

\section{A. Weighted quantization of state and input space}

Quantization is a major task in qualitative control. In most of systems in far states conditions, the resolution of state space splitting can be very low so that the system only knows that its states are far from the set point so that it acts with the highest saturated input to compensate the errors. Therefore, we can partition the far states in state space in wide sense. On the other hand, in the near state conditions, the system must converge to final state with sufficient accuracy. So the state space in these regions must be fine partitioned. The quantization of the input(s) is depends on the control strategy or the type of the driving system. In figure 1 there is an example of this quantization for a typical system [9]. In this example, the states of the second order system are $X_{1}$ and $X_{2}$. In general case the system can be of higher order and nonlinear. In these cases, the state space of the system should be non-uniformly partitioned.

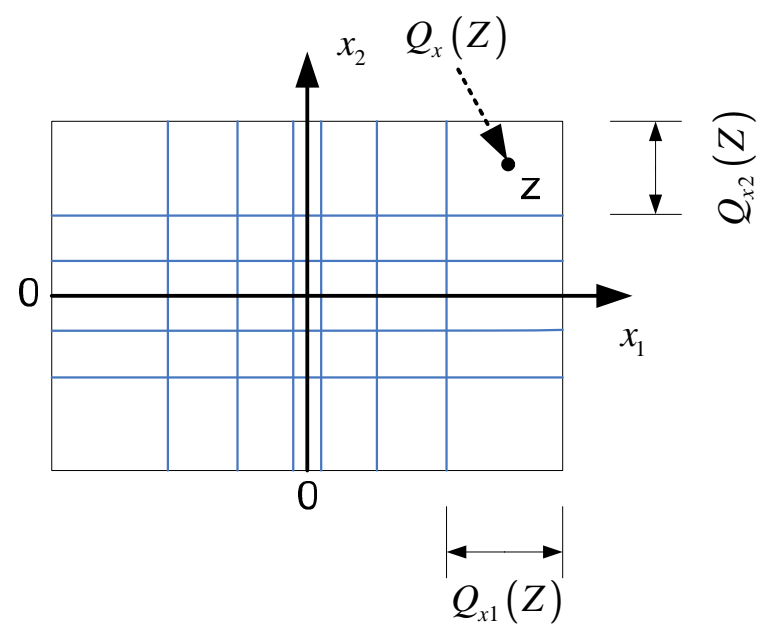

Fig.1 state space non-uniform quantization of a dynamic state system.

Each part of the states is numbered and used as a node or state in the overall graph of the system. Now the transition of each node or state must be evaluated.

\section{B. Monte-Carlo State transition calculation}

State transition is the most important part of the qualitative system identification. If the system is in state (node $i$ ) on time $t_{0}$, State transition indicates the state on time $t_{0}+T_{s}$ in the application of special quantize input. $T_{s}$ is the sample time of discrete time quantized system. Each quantized state or node for example in figure 1 is a square region in state space but the map of this region is not necessarily a square region and it may overlap with many state regions as shown in figure 2.

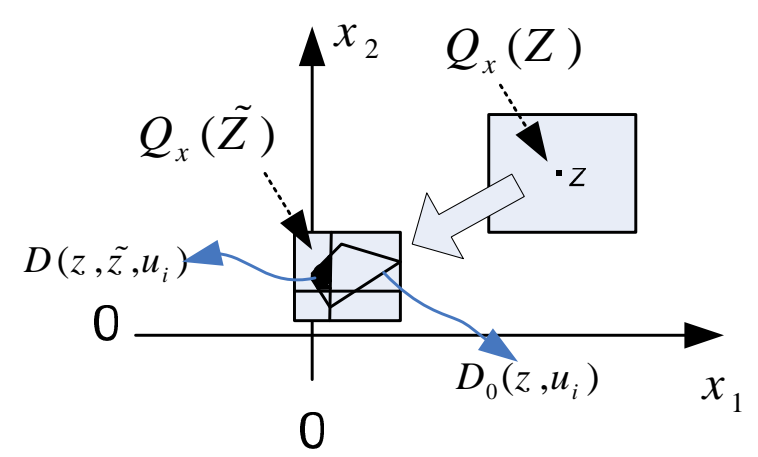

Fig. 2 State transition of a dynamic discrete event system, when $u_{i}$ is as an input to the system.

To find out the overall transition states, a statistic method must be used. General state space equation is:

$x\left[(k+1) T_{s}\right]=f\left(x\left[k T_{s}\right], u\left[k T_{s}\right]\right)$

The quantized states or nodes are defined as in figure 1:

$Q_{x i}\left(z_{j}\right)=\left\{x_{i} \mid g_{j} \leq x_{i} \leq g_{j+1}\right\}$

where, $g_{i}$ is the edge of each region in quantization.

In n-dimension system, we have the quantized state or node as:

$Q_{x}(z)=Q_{x 1}(z) \otimes Q_{x 2}(z) \otimes \ldots \otimes Q_{x n}(z)$

As it is clear in figure $2, \mathrm{z}$ state $\left(Q_{x}(z)\right)$ is mapped to more than one state and we can weigh the transition of the state transition diagram, proportional to the probability of the accruing of next state. In figure $2, D_{0}\left(z, u_{i}\right)$ is the map of the primary state, $z$, and $D\left(z, \tilde{z}, u_{i}\right)$ is the intersection of $D_{0}\left(z, u_{i}\right)$ and the node $\tilde{z}$.The transition probability is defined as:

$$
p\left(z, \tilde{z}, u_{i}\right)=\frac{\int_{D\left(z, \tilde{z}, u_{i}\right)} d x}{\int_{D_{0}\left(z, u_{i}\right)} d x}
$$

In nonlinear and complicated dynamic systems, $D_{0}\left(z, u_{i}\right)$ cannot be evaluated analytically. The integrations in this formula are very complicated due to the nonlinear and perhaps stochastic behavior of the system model. Therefore, in (4), Monte Carlo statistic integration method is used for the integration. A complicated program with a suitable GUI is used as in figure 3 to generally quantize and analyze a dynamical system of higher order. 

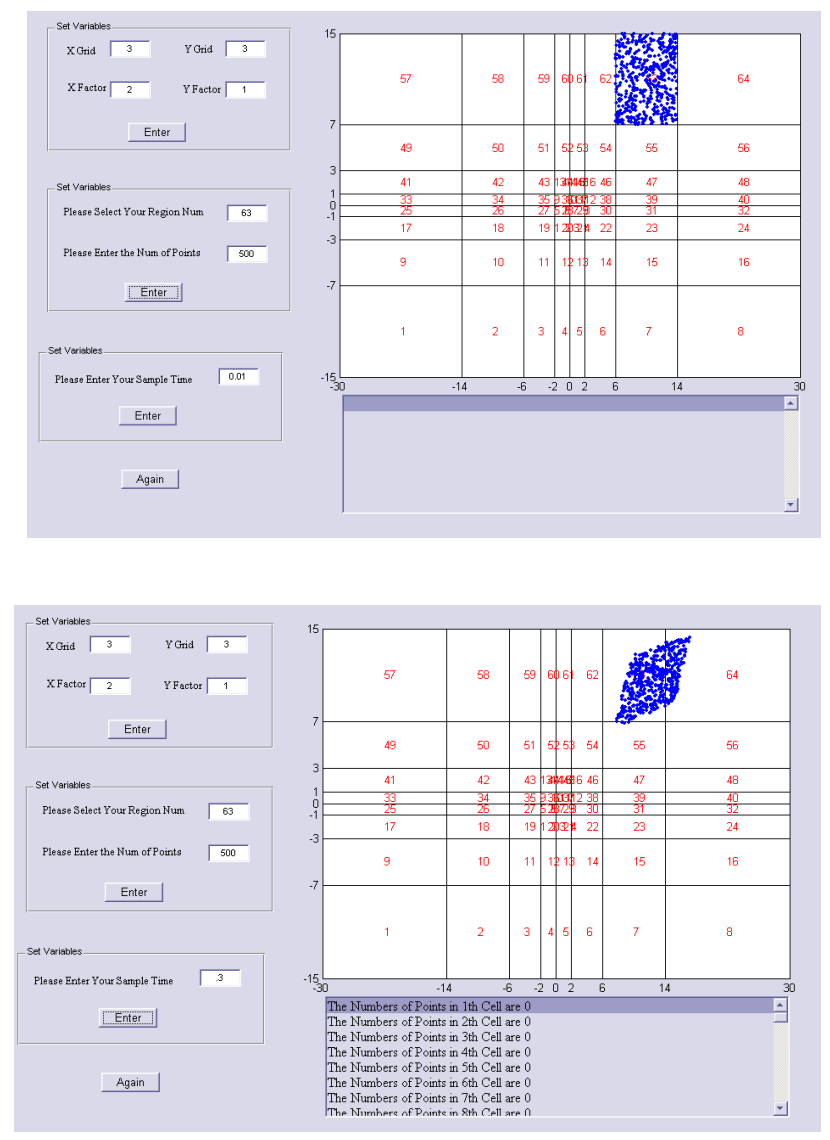

Fig. 3 GUI for evaluating the state transition of a dynamic discrete event system

\section{Monte-Carlo algorithm}

Monte- Carlo algorithm is a statistic method. It can be used off-line for evaluation of (4). This algorithm is summarized as follow [9]:

1. Point generation in $Q_{x}(z)$ domain. These points are distributed uniformly in the region and quantity of them, must be much enough for good estimation of the integrals in (4).

2. Application of the system map of eq. (1) on the pre-defined points.

3. Counting the mapped points in each of the $Q_{x}(\tilde{z})$ regions and divide them by the total number of points.

The results are yield by the calculation of $p\left(z, \tilde{z}, u_{i}\right)$.

This procedure must calculate for each $u_{i}$.

\section{GRAPH REPRESENTATION OF DYNAMIC SYSTEM}

The result of the algorithm of section II is a complicated table that can be interpreted as a graph. In this graph, each quantized region in state space is a node and branches between nodes represent the possibility of transient of the former state to the proceeding one. On each branch, the input that cased this transient and its probability of occurrence is labeled [7]. This theme is sketched typically in figure 4. This graph estimates the dynamic behavior of the system.

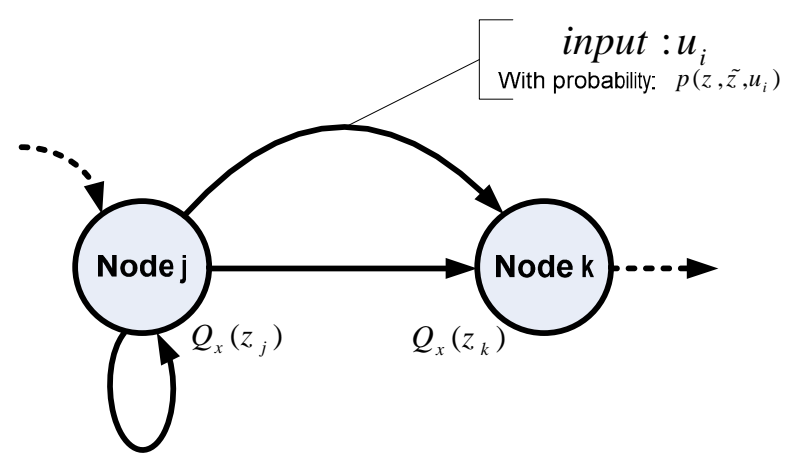

Fig. 4 Graph representation of dynamic system

\section{CONTROL RULE GENERATION WITH ANT COLONY SYSTEM (ACS)}

\section{A. Control strategy}

The next step is to find out a control strategy from the graph representation of the system. This control effort is in a specified set of inputs that are quantized. We have:

$$
[u(k)]=g([x(k)])
$$

where, [.] represents the quantized value. The control law, $g(\cdot)$ should be selected to maximize the probability of reaching final state that is $Z=0$, in minimum time. It is clear from the Graph representation of dynamic system that each node transition has a specified probability with the defined quantized input value. The main task is to reach zero state in minimum time. Therefore, in each node, an input with the highest probability in overall system to reach the zero state should be specified. The problem has complicated constraints that are to follow the dynamic of the proposed system. This complicates the optimization problem and heuristic methods are suggested to solve the problem. The objective function is the following equation:

Maximize:

$$
\begin{aligned}
& \lim _{k \rightarrow \infty} . \text { prob. }\{[x(k)]=0\} \\
& \text { subject to: } \\
& \quad x(k+1)=f(x(k), u(k))
\end{aligned}
$$

\section{B. Ant colony system(ACS) algorithm as an efficient optimization method}

The described optimization problem is to find an optimal path in a complicated graph. Ant colony system (ACS) seems to be a suitable optimization method to solve this problem due to behavior of the ants. Ant colony optimization is a new idea that has recently been proposed for complicated optimization problems. This method is based on leaving pheromones and imitating ants' behaviors in the real world. Improvements in solving many problems such as the Traveling Salesman's Problem (TSP), the job Scheduling, Graph coloring, and routing in computer networks have been made by observing and modeling real ant colonies. The results of the optimization problems that are obtained by ant colony algorithms are as well as those obtain by other 
heuristic methods [16], [17], and [18].

Real ants have the capability of finding the shortest path from a food source to their nest by exploiting pheromone information and without using visual cues. Ants deposit pheromone on the ground, and follow the highest density of pheromone that is previously deposited by other ants. A way ants exploit pheromone to find a shortest path between two points is described in many articles such as [12]. The algorithm in which a set of artificial ants cooperate to the solution of a problem by exchanging information via pheromone deposited on graph edges, is inspired ant system. Ant system has been applied to combinatorial optimization problems like the traveling salesman problem (TSP) [11].

In ant system, each ant produces a complete tour by choosing the nodes according to a probabilistic state transition rule. This state transition rule is that the Ants prefer to move to nodes, which are connected by short edges with a high amount of pheromone. A global pheromone-updating rule is applied once all ants have completed their tours. A fraction of the pheromone evaporates on all edges and then each ant deposits an amount of pheromone on edges, which is proportion to how short its tour was. Edges, which belong to many short tours, are the edges that receive the greater amount of pheromone. The process is then iterated.

A random-proportional rule that is the state transition rule used by ant system is given by equation (7). It gives the probability with which ant $\mathrm{k}$ in node $\mathrm{r}$ chooses to move to the node $\mathrm{s}$.

$$
p_{k}(r, s)=\left\{\begin{array}{cc}
\frac{[\sigma(r, s)][\eta(r, s)]^{\beta}}{\sum_{u \in J_{k}(r)}[\sigma(r, s)][\eta(r, s)]^{\beta}} & s \in J_{k}(r) \\
0 & \text { Otherwise }
\end{array}\right.
$$

In this equation $\sigma$ is the pheromone, $\eta=1 / \delta$ is the inverse of the distance $\delta(r, s), J_{k}(r)$ is the set of nodes that remain to be visited by ant $\mathrm{k}$ positioned on node $\mathrm{r}$. The relative importance of pheromone versus distance is $\beta$ and it is always positive $(\beta>0)$ to make the solution feasible. In Eq. (7), we multiply the corresponding heuristic value $\eta(r, s)$ by the pheromone on edge $(r, s)$. In this way, we favor the choice of edges which have a greater amount of pheromone and which are shorter. In ant system, the global pheromone-updating rule is implemented as follows. Pheromone is updated on all edges according to the following rule while all ants have built their tours.

$$
\begin{aligned}
& \sigma(r, s) \leftarrow(1-\alpha) \sigma(r, s)+\sum_{k=1}^{m} \Delta \sigma_{k}(r, s) \\
& \Delta \sigma_{k}(r, s)=\left\{\begin{array}{cc}
1 / L_{k} & \text { if }(r, s) \in \text { tour of ant k } \\
0 & \text { otherwise }
\end{array}\right.
\end{aligned}
$$

$0 \leq \alpha \leq 1$ is a pheromone decay parameter, $m$ is the number of ants, and $L_{k}$ is the length of the tour performed by ant k. Greater amount of pheromone allocated to shorter tours by pheromone updating rule. The pheromone updating formula was meant to simulate the change for pheromone due to both the pheromone evaporation, and to addition of new pheromone deposited by ants on the visited edges.

Pheromone placed on the edges plays the role of a distributed long-term memory: This memory is not stored locally within the individual ants, but is distributed on the edges of the graph. This allows an indirect form of communication.

We use this algorithm to find a path in the proposed graph of the system of pendulum, which is stable and converges to node zero in shortest time.

\section{PRESENTATION OF PROPOSED ALGORITHM}

The algorithm is summarized as in figure 5. Levels of the state space and input space quantization are the manual inputs of the algorithm. This information is the only thing that is provided by the expert person knowledge to the algorithm. The speed of the convergence process and the resolution of the final output are the major information for the quantization of the state and input level settings.

In the algorithm, the graph modeling of the dynamical system is completed when the state transition probability of each node $\left(n_{j}\right)$, to the other node $\left(n_{k}\right)$, with the application of the input $\left(u_{i}\right)$, is calculated. In the next step, the best input for each node is calculated by aim of ACS optimization process. The overall result is a Look Up Table (LUT), that may be used in controlling the system. In this LUT, an optimum input is assigned to each node or state space zone.

LUT is implemented in the hardware of the system very easily. The structure of the qualitative control process permits the use of simple and non-expensive types of sensors. Therefore, the control strategy may be implemented in a very simple manner. The next section belongs to the implementation of the Qualitative optimal process to a complicated dynamical system and shows the effectiveness of the described strategy.

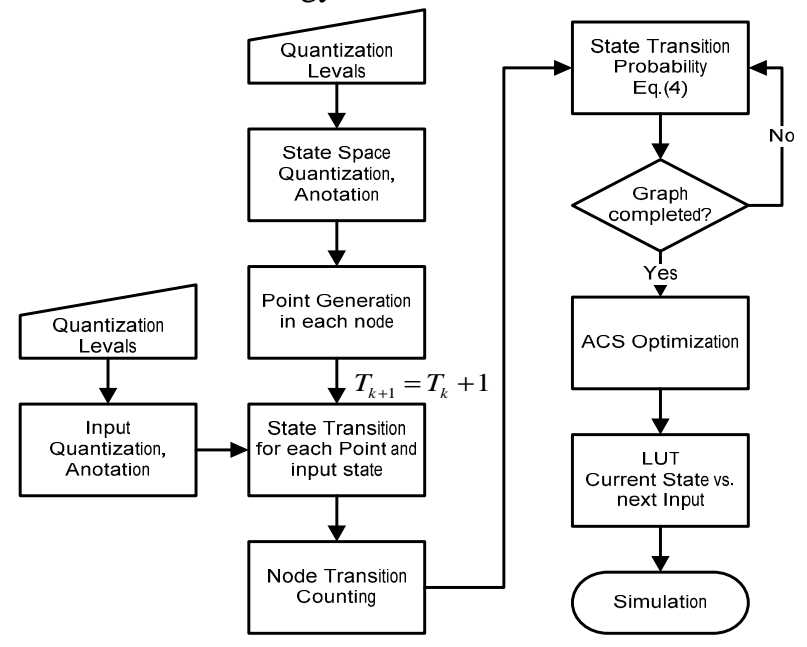

Fig.5 Proposed algorithm

\section{2DOF INVERTED PENDULUM EXAMPLE}

\section{A. Model description}

The problem of balancing a two-degree of freedom (DOF) pendulum has been a benchmark problem in demonstrating 
and motivating various control design techniques [1], [2]. From a control design perspective, nonlinearity and open loop system unstability are discussed as challenge problems. In addition, the need for a sensor system to measure the inclination angles of the pendulum contribute to the complexity of the balancing problem. A spherical pendulum system based on an $X-Y$ table was designed and constructed. Then, a control model was developed by projecting the system onto the $\mathrm{x}-\mathrm{z}$ and $\mathrm{y}-\mathrm{z}$ planes of the inertial coordinate system. These projections were treated independently and were controlled individually by the $\mathrm{x}$ - axis and $\mathrm{y}$ - axis, respectively. Two stabilization controllers were designed for each planar inverted pendulum. In figure 5 and 6 , the schematic and picture of the system is presented. The $X-Y$ table is equipped with special DC motors as actuator, the pendulum is fixed in a cart, and it can rotate free in all directions. The sensors of angles are a track ball mouse.

Actually, different friction terms should be added into the dynamics model in a practical implementation. Two friction terms are considered in our model, one is the viscous friction when carriage $\mathrm{M}$ performs its linear motion on the ball screw; the other is the viscous friction when the pendulum rotates around its pivot. Let the modified dynamics model of a spherical inverted pendulum as following:

$$
\begin{aligned}
& \left(M_{x}+m\right) x^{\prime \prime}+\frac{1}{2} m l \alpha^{\prime \prime} \operatorname{Cos} \alpha-\frac{1}{2} m l \alpha^{\prime \prime 2} \operatorname{Sin} \alpha+c_{M x} x^{\prime}=\tau_{x} \\
& \frac{1}{2} m l x^{\prime \prime} \operatorname{Cos} \alpha+\frac{1}{3} m l^{2} \alpha^{\prime \prime}+c_{m x} \alpha^{\prime}-\frac{1}{2} m g l \operatorname{Sin} \alpha=0 \\
& \left(M_{y}+m\right) y^{\prime \prime}+\frac{1}{2} m l \beta^{\prime \prime} \operatorname{Cos} \beta-\frac{1}{2} m l \beta^{\prime \prime 2} \operatorname{Sin} \beta+c_{M y} y^{\prime}=\tau_{y} \\
& \frac{1}{2} m l y^{\prime \prime} \operatorname{Cos} \beta+\frac{1}{3} m l^{2} \beta^{\prime \prime}+c_{m y} \beta^{\prime}-\frac{1}{2} m g l \operatorname{Sin} \beta=0
\end{aligned}
$$

\begin{tabular}{|c|c|}
\hline Symbol & Quantity \\
\hline$M_{x}$ & Mass of carriage respective to $x$-axis \\
\hline$M_{y}$ & Mass of carriage respective to y-axis \\
\hline$m$ & Mass of pendulum \\
\hline$l$ & Length of pendulum \\
\hline$x$ & Linear displacement of carriage in $\mathrm{X}$ direction \\
\hline$y$ & Linear displacement of carriage in $Y$ direction \\
\hline$\alpha$ & Inclination angle of pendulum on $x$-z projection \\
\hline$\beta$ & Inclination angle of pendulum on $y$-z projection \\
\hline$c_{M x}, c_{M y}$ & $\begin{array}{l}\text { Viscous friction coefficient for motion of } \\
\text { carriage in } X \text { and } Y \text { directions, respectively }\end{array}$ \\
\hline$c_{m x}, c_{m y}$ & $\begin{array}{l}\text { Viscous friction coefficient for rotary motion of } \\
\text { pendulum on } x \text {-z and } y-z \text { projections, } \\
\text { respectively }\end{array}$ \\
\hline$\tau_{x}, \tau_{y}$ & $\begin{array}{l}\text { Force provided by the motors to implement } \\
\text { linear motion in } X \text { and } Y \text { direction, respectively }\end{array}$ \\
\hline
\end{tabular}

where, the following abbreviations are defined as in table1.

TABLE 1 INPUT QUANTIZATION TABLE

\section{B. Practical System}

The performance of the experimental hardware is very important part of this project. The reliability of the sensor system and the flexible motion of the $X-Y$ table and were the decisive factors in the success of the whole system [10]. Figure 6 is a schematic description of the overall system. Moreover, the practical system is shown in figure 7. A re-constructed trackball system is proposed as angle sensor in the system as shown in figure 8 . The control box is FPGA (Xilinx Spartan XC2S100PQ208) based and designed to negotiate with windows XP based software by USB2 port via FT245. The sensors of pendulum are two digital encoders in a track ball mouse. The actuators that produce forces are small DC motors that are coupled to the system. Current feedback compensation is implemented in DC driver systems. While the armature current is proportional to the applied torque in the DC motor, the torque reference is the command input to the drive system. Therefore the inputs to the $X-Y$ table are Forces provided by the motors to implement linear motion in $X$ and $Y$ direction, i.e. $\tau_{x}$ and $\tau_{y}$ respectively. These forces are proportional to the direct motor torques, $\hat{\tau}_{x}$ and $\hat{\tau}_{y}$. The

\begin{tabular}{|c|c|c|c|c|c|}
\hline $\begin{array}{c}\text { Input } \\
\text { Levels }\end{array}$ & $\hat{\tau}_{x(-H)}$ & $\hat{\tau}_{x(-L)}$ & $\hat{\tau}_{x(0)}$ & $\hat{\tau}_{x(+L)}$ & $\hat{\tau}_{x(+H)}$ \\
\hline $\begin{array}{c}\text { Annotati } \\
\text { on }\end{array}$ & $\hat{\tau}_{y(-H)}$ & $\hat{\tau}_{y(-L)}$ & $\hat{\tau}_{y(0)}$ & $\hat{\tau}_{y(+L)}$ & $\hat{\tau}_{y(+H)}$ \\
\hline $\begin{array}{c}\text { Torque } \\
\text { level }\end{array}$ & $\begin{array}{c}-0.3 \\
\text { (N.m.) }\end{array}$ & $\begin{array}{c}-0.08 \\
(\mathrm{~N} . \mathrm{m} .)\end{array}$ & $\begin{array}{c}0 \\
\text { (N.m.) }\end{array}$ & $\begin{array}{c}0.08 \\
\text { (N.m.) }\end{array}$ & $\begin{array}{c}-0.3 \\
\text { (N.m.) }\end{array}$ \\
\hline
\end{tabular}
specified inputs are direct DC motor torques and are partitioned to specified levels as in table 2 .

TABLE 2 INPUT QUANTIZATION TABLE

These Torque command levels are selected by the expert knowledge.

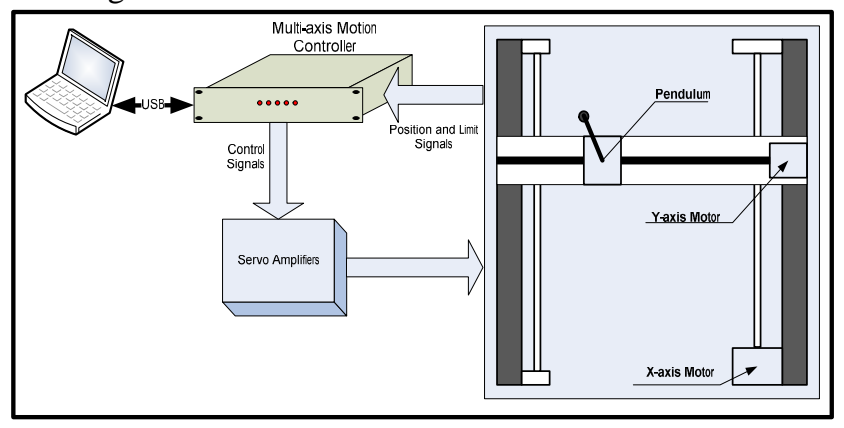

Fig.6 schematic of X-Y table system for experiments of spherical inverted pendulum

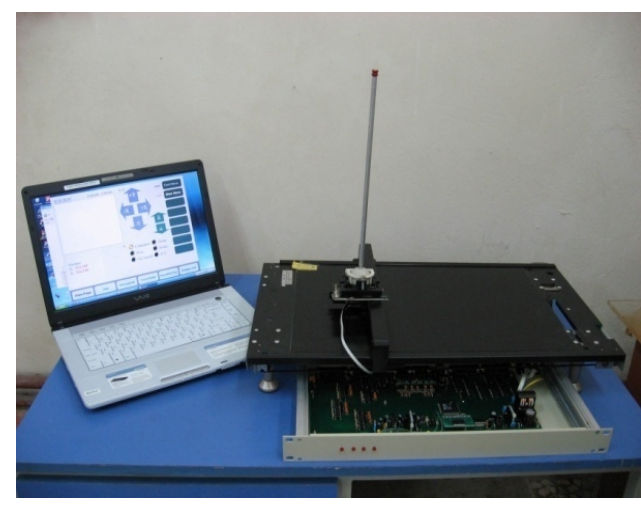

Fig.7 A view of of operating 2DOF inverted pendulum 
Fig. 8 A trackball that is re-constructed as $\alpha-\beta$ incremental encoder-type $r \mid$
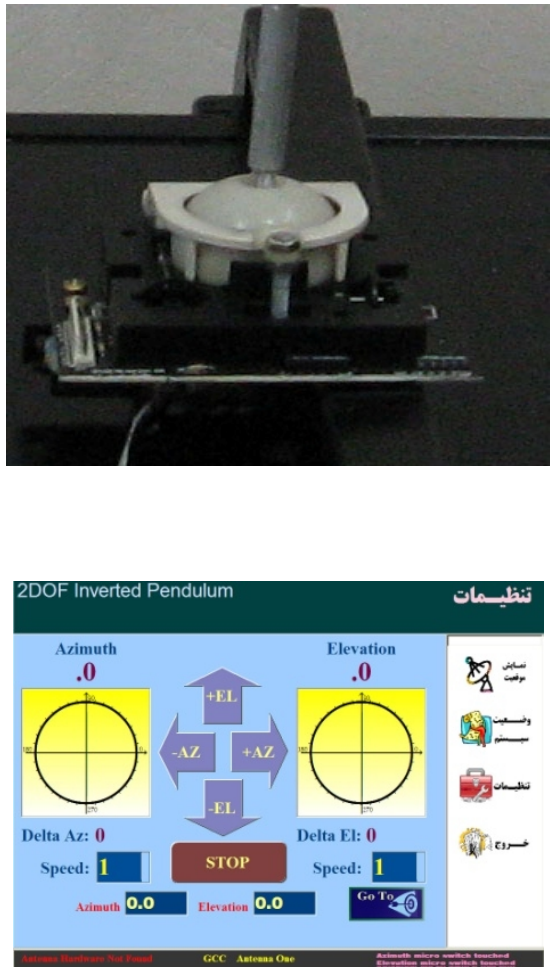

Fig.9 mnemonic operating menu of 2DOF inverted pendulum control software

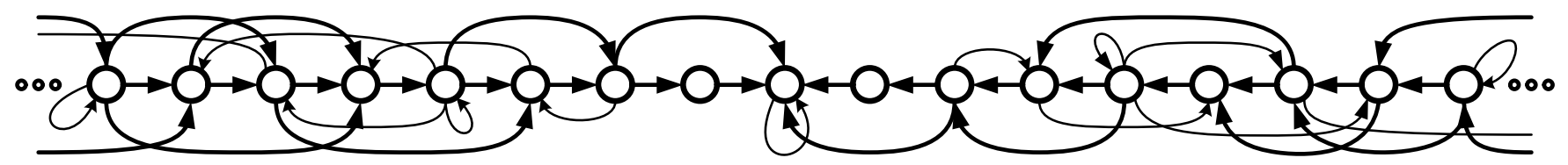

Fig.10 Portion of system graph 


\section{Practical Results}

As it is indicated earlier, around the set point, $X$ and $Y$-axis are approximately independent and the system converted to two one-dimensional inverted pendulums. First, three-dimensional $\left(\alpha, \alpha^{\prime}, x\right)$ space and $\left(\beta, \beta^{\prime}, y\right)$ space, and the inputs, $\tau_{x}$ and $\tau_{y}$ must be partitioned and quantized properly. Each set of equations can be converted to a special graph. The first one is sketched in figure 10. In the next step, the optimized path with minimum travel time with the ant colony system is obtained. This path defines the proper inputs, the application of them to actuators, are done, and the results are presented in figure 11 and 12. The resolution of response depends on the quantization process and that how fine we define the near final set point parameters and partition levels.

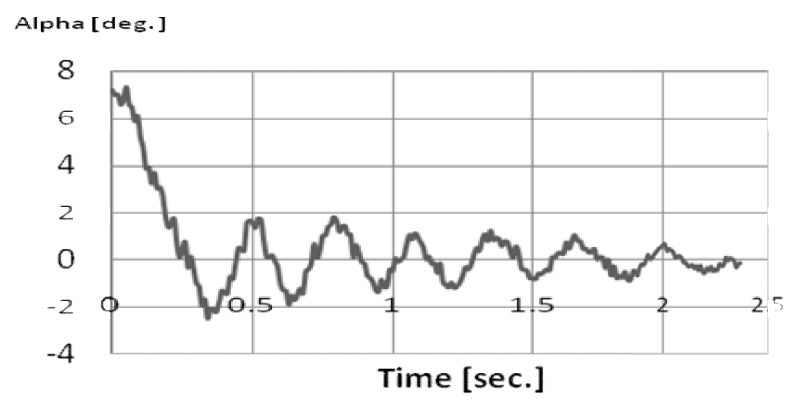

Fig.11 alpha Resulted from the qualitative control

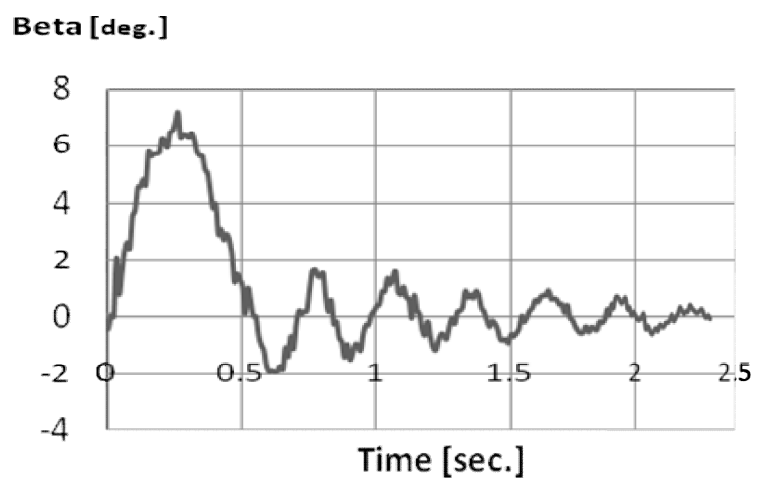

Fig.12 beta Resulted from the qualitative control

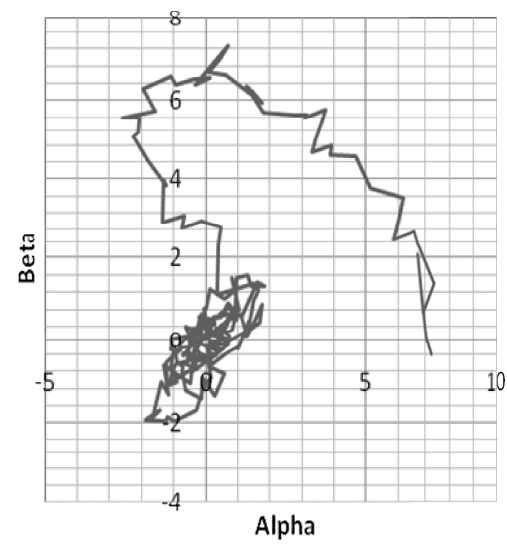

Fig.13 moving trajectory of pendulum

\section{CONCLUSION}

In this paper, a new optimal Qualitative controller was developed and implemented in real time for the first time for the precise position control of the 2DOF-inverted pendulum. The proposed controller is based on the variable level quantization of the system model state and input and identification of system as a complicated graph.

Moreover, a heuristic method compatible for optimization of such optimal routing system is proposed and implemented. The ant colony system (ACS) is suitable way of finding the shortest stable path to the origin state. The effectiveness of the method is proved by implementing the process in a complicated dynamical system i.e. inverted pendulum. This system is a good platform for evaluating the described method due to its strongly unstable nature.

\section{REFERENCES}

[1] K.J. Åström, K. Furuta" Swinging up a Pendulum by Energy Control" Proceeding of 13th IFAC World Congress, San Francisco, CA, 1996.

[2] D. Angeli, " Almost global stabilization of the inverted pendulum via continuous state feedback" Automatica 37, 1103-1108, 2001

[3] A. Rezazade, A. Sayyah, M. Aflaki, "Optimization of THD and Suppressing Certain Order Harmonics in PWM inverters using Genetic Algorithms, " IEEE International Symposium on Intelligent Control (ISIC) October 4 to October 6, Munich, Germany, 2006.

[4] A. Sayyah, M. Aflaki, A. Rezazade "GA-Based Optimization of Total Harmonic Current Distortion and Suppression of Chosen Harmonics in Induction Motors, " SPEEDAM SYMPOSIUM 23-26 May - Taormina (Italy), 2006.

[5] A. Rezazade, A. Sayyah, M. Aflaki” Optimal PWM for Minimization of Total Harmonic Current Distortion in High-Power Induction Motors using Genetic Algorithms, " SICE - ICASE International Joint Conference EXCO (Bussan Exhibition \& Convention Center), Bussan, KOREA, 2006.

[6] A. Rezazade, A. Sayyah, M. Aflaki” Modulation Error Observation and Regulation for use in Off-line Optimal PWM Fed High Power Synchronous Motors, "1st IEEE Conference on Industrial Electronics and Applications (ICIEA 2006), Singapore on 24 - 26 May, 2006.

[7] G. Lichtenberg, J. Lunze, R. Scheuring, J. Schroder" Prozessdiagnose mittlels qualitativer Modelle am Beispiel eines Wasser stoffverdichters, "Automatisierungstechnik 47, 101-109, 1999.

[8] [8] J. Lunze" Qualitative modeling of linear dynamical systems with quantized state measurements" Automatica 30, 417-43, 1994.

[9] A. Rezazade, P. Jabedar, " Qualitative rule base generator and its application in DC motor control" Electrical engineering international conference, Tehran, Iran.

[10] Yang Rong, " Geometric Techniques for Control of a 2-DOF Spherical Inverted Pendulum" Ph.D. thesis Hong Kong University of Science and Technology, 2003.

[11] M. Dorigo, L. Gambardella, "Ant Colony System:A Cooperative Learning Approach to theTraveling Salesman Problem" IEEE Transactions on Evolutionary Computation, Vol.1, No.1, 1997.

[12] R. Beckers, J.L. Deneubourg, and S. Goss, "Trails and U-turns in the selection of the shortest path by the ant Lasius Niger, "Journal of Theoretical Biology, vol. 159, pp. 397-415, 1992.

[13] G. Conte, C.H. Moog and A.M. Perdon, "Algebraic Methods for Nonlinear Control Systems. Theory and Applications, " Springer-Verlag, 2006.

[14] H. Maurer, C. B"uskens, J.-H.R. Kim and C.Y.Kaya, “ Optimization methods for the verification of second order sufficient conditions for bang-bang controls, “[13], vol. 26, 129-156, 2005.

[15] H. N'emeth, K. M. Hangos, " Nonlinear control of an electro-pneumatic rotection valve for circuit pressure limiting, " Research Report of Computer and Automation Research Institute, Budapest, Hungary, SCL-2/2003.

[16] K.M. Sim, W. H. Sun, “ Ant colony Optimization for routing and load balancing: survey and new directions, " IEEE trans. Systems, man, and Cybenetics, vol. 33, no.5, pp. 560-572, 2003.

[17] L. Yong, Guang zhou, Z. Fan jan, L. Sand Xiao run, “ Adaptive swarm based routing in communication networks, " Journal of Zhejiang University Science, no.5, pp. 867-872, 2004. 
[18] S. Doi, M. Yamamura, “ Congestion Detection and Clearing History of Trip Time in AntNet" IEEE congress on Evalutionary Computation CEC, vol.2, pp.1621-1628, 2005

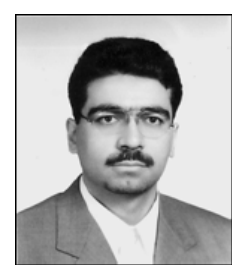

A.Rezazade was born in Tehran, Iran in 1969. He received his B.S. and M.S. degrees and Ph.D. from Tehran University in 1991, 1993, and 2000, respectively, all in electrical engineering. He has two years of research in Electrical Machines and Drives laboratory of Wuppertal University, Germany, with the DAAD scholarship during his Ph.D. and Since 2000 he was the head of CNC EDM Wirecut machine research and manufacturing center in Pishraneh company. His research interests include application of computer controlled AC motors and EDM $\mathrm{CNC}$ machines and computer controlled switching power supplies. Dr. Rezazade currently is an assistant professor in the Power Engineering Faculty of Shahid Beheshti University. 